\title{
Are the Polyomaviruses BK and JC Associated with Opportunistic Infections, Graft-versus-Host Disease, or Worse Outcomes in Adult Patients Receiving Their First Allogeneic Stem Cell Transplantation with Low-Dose Alemtuzumab?
}

\author{
Laila Schneidewind $^{\mathrm{a}}$ Thomas Neumann $^{\mathrm{b}} \quad$ Florian Knoll $^{\mathrm{b}}$ Kathrin Zimmermann ${ }^{\mathrm{c}}$ \\ Sigrun Smola ${ }^{a}$ Christian Andreas Schmidt ${ }^{b}$ William Krüger ${ }^{b}$

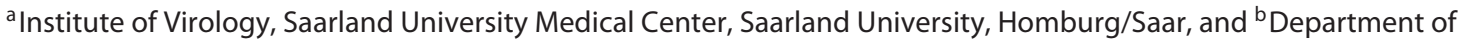 \\ Haematology/Oncology, and ' Friedrich Loeffler Institute of Medical Microbiology, University Medicine Greifswald, \\ Greifswald, Germany
}

For editorial comment see p. 1

\section{Keywords}

Adult allogeneic stem cell transplantation · BK-associated hemorrhagic cystitis · BK polyomavirus · Graft-versus-host disease $\cdot$ Transplantation-associated infections

\begin{abstract}
Background: The association of polyomaviruses BK and JC with other opportunistic infections and graft-versus-host disease (GvHD) in allogeneic stem cell transplantation is controversially discussed. Methods: We conducted a retrospective study of 64 adult patients who received their first allogeneic stem cell transplantation between March 2010 and December 2014; the follow-up time was 2 years. Results: Acute leukemia was the most frequent underlying disease (45.3\%), and conditioning included myeloablative (67.2\%) and nonmyeloablative protocols (32.8\%). All patients received $10 \mathrm{mg}$ of alemtuzumab on day -2 ( $20 \mathrm{mg}$ in case of mismatch) as GvHD prophylaxis. Twenty-seven patients (41.5\%) developed cytomegalovirus (CMV) reactivation. BKPyV-associated hemorrhagic cystitis was diagnosed in 10 patients (15.6\%). Other opportunistic infections caused by viruses or protozoa occurred rarely $(<10 \%)$. There was no association of BKPyV
\end{abstract}

\section{KARGER}

(C) 2017 S. Karger AG, Basel

E-Mail karger@karger.com

www.karger.com/aha or JCPyV with CMV reactivation, Epstein-Barr virus reactivation, human herpes virus 6 , or parvovirus B19 infection requiring treatment. There was a significant correlation of BKPyV-associated hemorrhagic cystitis with toxoplasmosis $(p=0.013)$. Additionally, there was a significant link of simultaneous BKPyV and JCPyV viruria with toxoplasmosis ( $p=$ 0.047). BKPyV and JCPyV were not associated with GvHD, relapse, or death. Conclusion: We found no association of BKPyV or JCPyV with viral infections or GVHD. Only the correlation of both polyomaviruses with toxoplasmosis was significant. This is a novel and interesting finding.

(c) 2017 S. Karger AG, Basel

\section{Introduction}

The polyomaviruses BK (BKPyV) and JC (JCPyV) may cause opportunistic infections in immunocompromised patients [1]. BK viruria occurs in up to $100 \%$ of stem celltransplanted patients and can lead to $\mathrm{BKPyV}$-associated hemorrhagic cystitis in up to $40 \%$ [2]. Less frequent in these patients are JC viruria and JCPyV-associated hemorrhagic cystitis, but JCPyV is still an important pathogen

Laila Schneidewind

Institute of Virology, Saarland University Medical Center Saarland University

Kirrberger Strasse 100, DE-66424 Homburg/Saar (Germany)

E-Mail laila.schneidewind@uks.eu 
since it can cause progressive multifocal leukoencephalopathy $[3,4]$. The most important point about these viral infections is that they can lead to severe morbidity in stem cell-transplanted patients. For example, in a retrospective study of allogeneic stem cell transplantation with 2,477 patients, BK viruria was significantly associated with a loss of kidney function and a worse overall survival [5]. Nearly the same results were described before by Gilis et al. [6] and the authors also placed emphasis on the high financial costs of treating this complication.

Different risk factors and other opportunistic infections associated with these viruses have been discussed controversial lately, but there is a lack of data for adult allogeneic stem cell transplantation. Several studies arise from kidney or solid organ and pediatric stem cell transplantation. Another problem are the heterogenic populations of these studies, as they include different conditioning regimes such as antithymoglobulin or alemtuzumab. Especially for alemtuzumab, dosing is also a major issue in terms of adverse events, including opportunistic infections or infectious complications [7]. To our knowledge there are no studies on low-dose alemtuzumab for graftversus-host disease (GvHD) prophylaxis in adult allogeneic stem cell transplantation and its association with the $\mathrm{BKPyV}$ or JCPyV. However, there are also sparse data for JCPyV in comparison to BKPyV. The correlation with other opportunistic infections like cytomegalovirus (CMV), human herpes virus 6 (HHV-6), or Epstein-Barr virus (EBV) and the association with GvHD are controversially discussed [8-11]. Since BKPyV and JCPyV are important pathogens in allogeneic stem cell transplantation, it is important to know the risk factors and associated infections. This knowledge is important to develop further prospective clinical trials and experimental studies for drug development, as no causal therapy has been established so far.

Therefore, we conducted a retrospective analysis of adult patients receiving their first allogeneic stem cell transplantation in a single institution (University Medicine Greifswald) uniformly using low-dose alemtuzumab as GvHD prophylaxis. The analyzed endpoints were the correlation of BKPyV and JCPyV with other transplantassociated opportunistic infections and acute or chronic GvHD.

\section{Materials and Methods}

Development of This Study and Study Population

This study was designed according to the guidelines in the synthesis of qualitative research (ENTREQ) (equatornetwork.org), an international initiative that provides robust reporting guidelines [12].

Data from patients' records, including demographic characteristics, patient history, and the results of blood and urine tests and other related tests in adult patients with hematological disease who underwent their first allogeneic stem cell transplantation from March 2011 to December 2014 at our institution were collected. The observation period was from the beginning of the conditioning therapy until 2 years after stem cell transplantation; outpatient data were included. We decided to start this study at that time since a quantitative PCR for BKPyV and JCPyV had been established in the Institute of Medical Microbiology at the University of Greifswald by then. For that time period we could identify 68 eligible patients, but we finally excluded 4 patients due to incomplete data.

Afterwards, a data bank of these patients was established using SPSS 23.0 software.

All of the procedures performed in this study were in accordance with the ethical standards of the institutional and/or national research committee and in compliance with the 1964 Helsinki Declaration and its later amendments or comparable ethical standards. For this type of study, formal consent was not required.

\section{Assessment of Infectious Complications}

$\mathrm{BKPyV}$ and JCPyV were assessed by quantitative PCR in urine using a commercially available kit (LightMix Polyoma JC-BK; Tib Molbiol, Germany) on clinical suspicion only (mainly hemorrhagic cystitis); no other material (i.e., blood samples) was analyzed for $\mathrm{BKPyV}$ and JCPyV. BKPyV-associated cystitis was defined as hemorrhagic cystitis with macrohematuria in the presence of $\mathrm{BKPyV}$ in urine.

CMV reactivation and urinary tract infections were assessed on a regular basis during in-house treatment. CMV was assessed in blood samples at least 2 times a week by quantitative PCR; UTI was assessed at least 1 time a week by midstream urine sampling and culture, both regardless of clinical signs or symptoms during the inpatient treatment. During outpatient treatment and follow-up, CMV-DNA was measured quantitatively by rt-PCR up to day +100 on every visit.

The other infectious pathogens like EBV, HHV-6, toxoplasmosis, and parvovirus B19 were assessed by PCR on clinical suspicion only (e.g., in case of fever, diarrhea, rash, or any other symptom compatible with an infection).

Preemptive antiviral treatment of CMV reactivation with ganciclovir or foscarnet was started in cases of 1,000 copies $/ \mathrm{mL}$. Treatment of all other infections or reactivations was initiated at the decision of the physician in charge.

\section{Definitions and Statistical Analysis}

Day 0 of the timeline was defined as the day of infusion of the stem cell product. Engraftment of leukocytes was the first of 3 consecutive days with a leukocyte count of at least $1 \times 10^{9} / \mathrm{L}$. In the same way, engraftment of thrombocytes was defined as the first of 3 consecutive days with a platelet count of at least $20 \times 10^{9} / \mathrm{L}$, followed by a transfusion-independent increase above $20 \times 10^{9} / \mathrm{L}$.

Only infections requiring treatment were included in our analysis. In our study, we defined CMV reactivation, EBV reactivation, HHV-6 and parvovirus B19 infection, or toxoplasmosis as an infection and/or reactivation requiring treatment (e.g., rituximab therapy in case of EBV reactivation). \begin{tabular}{ll}
\hline & Acta Haematol 2017;138:3-9 \\
DOI: $10.1159 / 000468972$
\end{tabular}
Schneidewind/Neumann/Knoll/

Zimmermann/Smola/Schmidt/Krüger 
For each numeric variable, the numeric distribution was preliminarily assessed via the Kolmogorov-Smirnov test; due to the small sample size, histograms were also used. Descriptive statistics were obtained (i.e., means \pm SD for normal distributions or with medians [range] for nonparametric data). The $\chi^{2}$ test (parametric) and the Mann-Whitney U test (nonparametric) were used for categorical and continuous variables, respectively. Survival data were assessed using the Kaplan-Meier method. All reported $p$ values were based on a 2 -sided hypothesis. $p<0.05$ was considered statistically significant.

All statistical calculations were performed using Statistical Package for the Social Sciences 23.0 software (SPSS Inc., Chicago, IL, USA).

\section{Results}

Demographic Characteristics of the Study Population

The baseline characteristics are shown in Table 1. We found a population as expected for adult allogeneic stem cell transplantation, with a median age of 52.0 years and acute myeloid leukemia as the most frequent disease $(n=$ $22 ; 34.4 \%)$. All patients received low-dose alemtuzumab (10 $\mathrm{mg}$ on day -2 ; respectively, $20 \mathrm{mg}$ in case of mismatch) for GvHD prophylaxis. Conditioning included myeloablative $(67.2 \%)$ and nonmyeloablative protocols (32.8\%).

\section{Transplantation Data}

Table 2 shows all of the relevant transplantation data including the inpatient treatment.

\section{$B K$ and JC Virus Infections during the Inpatient}

\section{Treatment Period}

During the inpatient treatment period BK viruria occurred in 22 patients (34.4\%), and 10 of those patients with viruria developed $\mathrm{BKPyV}$-associated hemorrhagic cystitis (15.6\%).

Only 9 patients (14.1\%) developed JC viruria, and 6 of those patients $(9.4 \%)$ had symptomatic JC viruria, meaning urogenital symptoms like dysuria, alguria, or macrohematuria at the time of JC viruria. Interestingly, JC viruria is significantly associated with urogenital symptoms $(p<0.001)$. Therefore, we defined these cases as JC virusassociated cystitis. Four patients (6.2\%) developed simultaneous BK and JC viruria.

\section{Bacterial Urinary Tract Infections during the Inpatient Treatment}

Ten patients (15.6\%) had a bacterial urinary tract infection defined as significant bacteriuria in midstream urine in the presence of urogenital symptoms. Escherich-
Table 1. Demographic characteristics of the study population $(n=64)$

$\begin{array}{lc}\text { Gender } & \\ \text { Male } & 38(59.4) \\ \text { Female } & 26(40.6) \\ \text { Hematological disease } & \\ \text { AML } & 22(34.4) \\ \text { NHL } & 18(28.2) \\ \text { ALL } & 8(12.5) \\ \text { MDS } & 7(10.9) \\ \text { CLL } & 2(3.1) \\ \text { CML } & 2(3.1) \\ \text { MPS } & 2(3.1) \\ \text { Acute biphenotypic leukemia } & 2(3.1) \\ \text { T-prolymphocytic leukemia } & 1(1.6) \\ \text { Age, years } & 52.0(20-72) \\ \text { Conditioning regime } & \\ \text { Bu/Cy } & 42(65.6) \\ \text { Flu/TBI } & 8(12.5) \\ \text { Treo/Flu } & 6(9.4) \\ \text { Bu/Flu } & 4(6.3) \\ \text { Flu/Cy } & 2(3.1) \\ \text { Flu/Mel } & 1(1.6) \\ \text { Cy/TBI } & 1(1.6)\end{array}$

Values are presented as numbers (\%) or medians (range). AML, acute myeloid leukemia; NHL, non-Hodgkin lymphoma; ALL, acute lymphatic leukemia; MDS, myelodysplastic syndrome; CLL, chronic lymphatic leukemia; CML, chronic myeloid leukemia; MPS, myeloproliferative syndrome; $\mathrm{Bu}$, busulfan; $\mathrm{Cy}$, cyclophosphamide; Flu, fludarabine; TBI, total bone irradiation; Treo, treosulfane; Mel, melphalan.

Table 2. Transplantation data of the study population $(n=64)$

$\begin{array}{lc}\text { Donor } & \\ \quad \text { Matched related donor } & 16(25.0) \\ \quad \text { Matched unrelated donor } & 27(42.2) \\ \quad \text { Mismatched unrelated donor } & 21(32.8) \\ \text { Duration of leukopenia, days } & 14.0(10-31) \\ \text { Duration of thrombocytopenia, days } & 10.0(0-48) \\ \text { Transfused packed red blood cells, } n & 7.1 \pm 5.9 \\ \text { Transfused packed platelets, } n & 3.9 \pm 4.5 \\ \text { Transfused fresh frozen plasma, } n & 0.5 \pm 2.6 \\ \text { Primary immunosuppression (day 1) } & \\ \quad \text { Cyclosporine A } & 44(68.8) \\ \quad \text { Cyclosporine A + mycophenolate mofetile } & 20(31.2) \\ \text { Neutropenic fever during inpatient treatment } & \\ \quad \text { No } & 22(34.4) \\ \quad \text { Yes } & 42(65.4) \\ \text { Duration of inpatient treatment, days } & 45.0(23-114)\end{array}$

Values are presented as numbers (\%), means $\pm \mathrm{SD}$, or medians (range). 
Table 3. Association between opportunistic infections needing treatment and BK and JC virus in the study population $(n=64)$

\begin{tabular}{llllll}
\hline Opportunistic infection & BK viruria & BK cystitis & JC viruria & JC cystitis & $\begin{array}{l}\text { Simultaneous BK } \\
\text { and JC viruria }\end{array}$ \\
\hline Epstein-Barr virus & 0.636 & 0.536 & 0.561 & 0.644 & 0.711 \\
Cytomegalovirus & 0.886 & 0.586 & 0.381 & 0.202 & 0.744 \\
Human herpes virus 6 & 0.636 & 0.536 & 0.561 & 0.644 & 0.711 \\
Toxoplasmosis & 0.228 & $\mathbf{0 . 0 1 3}$ & 0.325 & 0.145 & $\mathbf{0 . 0 4 7}$ \\
Parvovirus B19 & 0.077 & 0.374 & 0.403 & 0.506 & 0.594 \\
\hline
\end{tabular}

Numbers are $p$ values. Bold values indicate statistical significance $(p<0.05)$.

Table 4. Association of acute and chronic GvHD with BK and JC virus in the study population $(n=64)$

\begin{tabular}{llllll}
\hline & BK viruria & BK cystitis & JC viruria & JC cystitis & $\begin{array}{l}\text { Simultaneous BK } \\
\text { and JC viruria }\end{array}$ \\
\hline Acute GvHD & 0.619 & 0.771 & 0.186 & 0.099 & 0.638 \\
Chronic GvHD & 0.783 & 0.316 & 0.691 & 0.454 & 0.548
\end{tabular}

Numbers are $p$ values. GvHD, graft-versus-host disease.

ia coli was the most frequent bacteria (80\%), followed by Enterococci (20\%).

We observed no association between bacterial urinary tract infection and BK viruria $(p=0.751)$ or BKPyV-associated hemorrhagic cystitis $(p=0.678)$. The same observation was made for JC viruria $(p=0.678)$ and JCPyVassociated cystitis $(p=0.941)$. When BK and JC viruria developed at the same time point, they were also not linked to bacterial urinary tract infection $(p=0.374)$.

Association of BK and JC Virus with the Occurrence of Neutropenic Fever during Inpatient Treatment

Neutropenic fever during inpatient treatment was not associated with BK viruria $(p=0.755)$ or $\mathrm{BKPyV}$-associated hemorrhagic cystitis $(p=0.683)$, nor was JC viruria $(p=0.408)$ and JCPyV-associated cystitis $(p=0.955)$ or the presence of $\mathrm{BK}$ and $\mathrm{JC}$ virus in urine at the same time $(p=0.683)$.

\section{Association of Opportunistic Infections Needing}

Treatment and BK and JC Virus

We analyzed the data for EBV, CMV, HHV-6, toxoplasmosis, and parvovirus B19 infection needing treatment until 2 years after transplantation. In this study pop- ulation EBV occurred 2 times (3.1\%), CMV reactivation 27 times (41.5\%), HHV-6 infection also 2 times (3.1\%), toxoplasmosis 3 times (4.6\%), and parvovirus B19 infection 4 times $(6.2 \%)$. Two of the 3 patients with toxoplasmosis also developed BK cystitis. The level of significance for each constellation is shown in Table 3. Interestingly, we only observed a significant correlation of BK cystitis or simultaneous BK and JC viruria with toxoplasmosis.

\section{Association of BK and JC Virus with GvHD}

Acute GvHD occurred in 23 patients (35.9\%), with skin as the most frequent manifestation location $(n=16$; $25 \%)$, followed by the bowel $(n=7 ; 10.9 \%)$ and the liver $(n=1 ; 1.6 \%)$. Slight complications (grade I and II GvHD) appeared 13 times (20.3\%) and severe adverse events (grade III and IV GvHD) 11 times (17.2\%). Chronic GvHD was observed in 5 patients $(7.8 \%)$, with a planned follow-up of 2 years after infusion of the stem cell product. Three patients had a skin manifestation (4.7\%) with limited disease and 2 patients a chronic GvHD of the bowel with extensive disease (3.1\%). The association of $\mathrm{BKPyV}$ and JCPyV with acute and chronic GvHD is shown in Table 4. $\begin{array}{ll}6 & \text { Acta Haematol 2017;138:3-9 } \\ \text { DOI: } 10.1159 / 000468972\end{array}$
Schneidewind/Neumann/Knoll/ Zimmermann/Smola/Schmidt/Krüger 
Table 5. Association of BK and JC virus with relapse or death in the study population $(n=64)$

\begin{tabular}{llllll}
\hline & BK viruria & BK cystitis & JC viruria & JC cystitis & $\begin{array}{l}\text { Simultaneous BK } \\
\text { and JC viruria }\end{array}$ \\
\hline Relapse & 0.381 & 0.981 & 0.798 & 0.839 & 0.833 \\
Death & 0.857 & 0.564 & 0.647 & 0.936 & 0.949 \\
\hline
\end{tabular}

Numbers are $p$ values.

\section{Association of BK and JC Virus with Outcome Data} and Survival

Since we analyzed the opportunistic infections and GvHD until 2 years after stem cell transplantation, we also show the survival data. The median survival time was 18.5 months, with a range of 1-65 months. At the time of reporting, 33 patients had died $(50.8 \%)$ and 19 patients had had a relapse $(29.2 \%)$. No association between the polyomaviruses $\mathrm{BK}$ and JC with relapse or death was found, and this is shown in Table 5. The Kaplan-Meier curve is shown in Figure 1.

\section{Discussion}

We conducted a retrospective study in a population of adult patients receiving their first allogeneic stem cell transplantation with low-dose alemtuzumab as GvHD prophylaxis, starting after established quantitative PCR for the polyomaviruses BK and JC. To our knowledge, this is the first study with a low-dose alemtuzumab stem cell transplantation population focused on BKPyV and JCPyV and their association with opportunistic infections and GvHD.

We must assume that this study still has a small sample size, but it is homogenous in terms of GvHD prophylaxis.

$\mathrm{BKPyV}$ - and JCPy-associated hemorrhagic cystitis were relatively rare in our population. For example, BK cystitis only occurred in $15.6 \%$ of cases despite literature references which describe this kind of cystitis in up to $40 \%$ of patients [2]. Infectious complications like CMV reactivation or toxoplasmosis are expected problems in these immunocompromised patients, but we found sparse adverse events in terms of these analyzed infections. Interestingly, although the question of whether $\mathrm{BKPyV}$ is associated with other typical transplantationrelated infections like CMV reactivation is controversially discussed, JCPy V has been less investigated. In their retrospective analysis of 339 patients who underwent alloge-

Association of BKV with Infection/GvHD

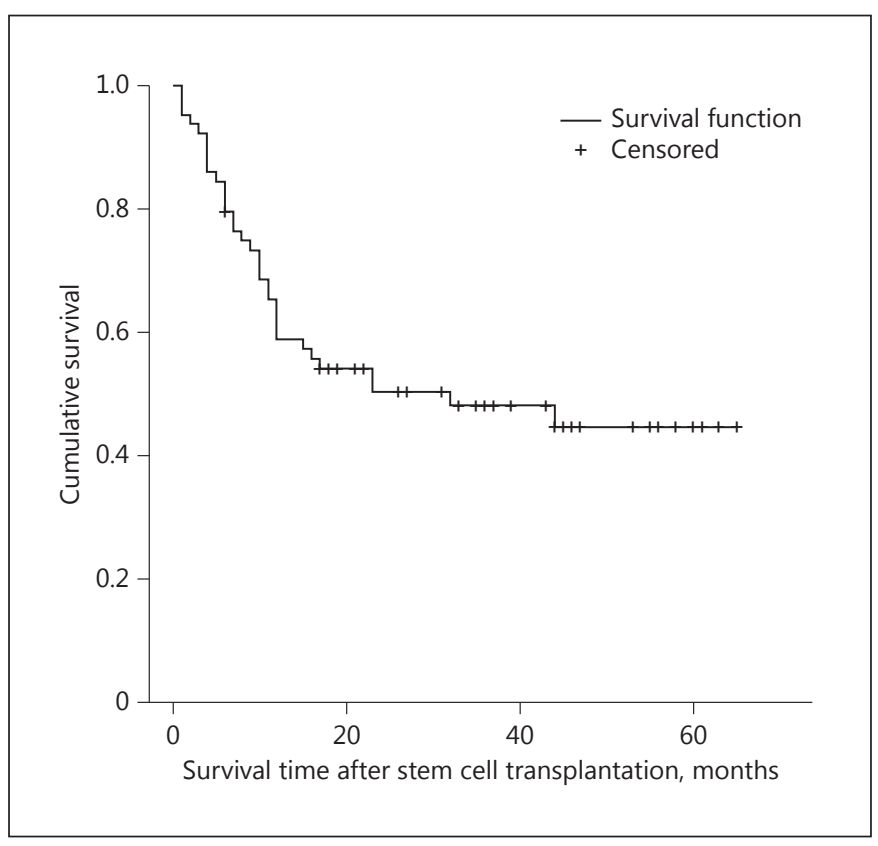

Fig. 1. Kaplan-Meier survival curve of the study population $(n=$ 64).

neic stem cell transplantation, Uhm et al. [13] described $\mathrm{CMV}$ viremia as a significant risk factor for $\mathrm{BKPyV}$-associated hemorrhagic cystitis, but another study with kidney-transplanted patients under immunosuppression identified CMV as a protecting factor against BKPyV infection $[13,14]$. We found no significant association of $\mathrm{BKPyV}$ and JCPyV with CMV in our patients. One reason for the controversial results described in several different studies might be the different patterns of immunosuppressive therapy also including alemtuzumab or antithymoglobulin; another problem is that most of those studies are retrospective. In our opinion, further prospective studies with exact drug monitoring and monitoring of the immune system, like T-cell counts, are necessary to

Acta Haematol 2017;138:3-9 DOI: $10.1159 / 000468972$ 
answer those questions. Furthermore, dosing of drugs like alemtuzumab is important. As we already mentioned, a big advantage of our analysis is the unified GvHD prophylaxis with low-dose alemtuzumab in the whole study population since a higher dose might trigger more infectious complications, as has been described before [7]. However, authors of studies with bigger samples sizes could argue that the data could not reach significance because of our small sample size, but with $p$ values ranging from 0.8 to 0.2 it is mathematically unlikely that statistical significance will be reached with a sample size of 300 . The same consideration could be made regarding the other analyzed subjects, especially GvHD, which will be discussed later.

Data for other transplant-associated infections are rare. Quintela et al. [8] reported that HHV-6 infection in allogeneic stem cell transplanted patients is significantly associated with CMV and BKPyV infections. To our knowledge, no data on JCPyV and other transplant-associated infections are currently available. Certainly, we cannot compare our study with the data of Quintela et al. [8] since the authors analyzed a very heterogenic population of stem cell-transplanted patients and the analysis was focused on chromosomal integration, viral coinfections, and T-cell reconstitution patterns.

Interestingly, we only found a significant correlation of BKPyV-associated hemorrhagic cystitis and simultaneous BK and JC viruria with toxoplasmosis requiring treatment. This is a novel finding. One explanation might be the high level of immunosuppression at the time when toxoplasmosis, BK, and JC viruria occurred but, to answer this question precisely, prospective data with drug and immune system monitoring are needed. Furthermore, it might only be a coincidence or maybe the polyomaviruses $\mathrm{BK}$ and JC require the same immune responses like toxoplasmosis, but there is a lack of data regarding this issue as well.

The data regarding $\mathrm{BKPyV}$ and the association with GvHD are controversial, but most analyses have found that BKPyV is associated with acute GvHD $[11,15]$. Interestingly, Peterson et al. [11] also performed a retrospective analysis in a homogenous population of adult allogeneic stem cell-transplanted patients receiving an FLAMSA-RIC protocol for conditioning and antithymoglobuline for GvHD prophylaxis and they found a significant association of BK viruria with acute GvHD. In comparison, our patients with no association with acute and chronic GvHD received low-dose alemtuzumab, but both studies are retrospective. As we mentioned before, a prospective evaluation with drug monitoring is neces- sary. Just as we stated for infections, reports about JCPyV and GvHD in the literature are rare, but in our population there was no correlation of JCPyV with GvHD.

One surprising result about $\mathrm{JCPyV}$ is that the virus is often associated with urological symptoms like dysuria even when there is only viruria without hematuria. This might not be important for patients' morbidity or mortality because there was also no link of the virus with relapse or death, but it may be relevant for patients' sense of comfort. Thus, we might have to consider a symptomatic therapy when the patient complains about symptoms like dysuria.

We found no association of $\mathrm{BKPyV}$ with relapse or death (meaning mortality), but BKPyV still might be important for patients' morbidity or worse outcomes, as we and others have reported before $[6,16]$.

Of course (as in every retrospective study with a small sample size) we have to assume that our data is biased, especially as data on BKPyV and JCPyV viruria were collected on patients with a clinical suspicion only.

Despite the mentioned limitations of our analysis, it also has advantages, like the homogenous study population in terms of GvHD prophylaxis with low-dose alemtuzumab, and the fact that it presents the first data on the association of BKPyV and JCPyV with toxoplasmosis and data on the absent association of both polyomaviruses with GvHD and EBV, CMV, HHV-6, and parvovirus B19.

On the whole, in our study of adult allogeneic stem cell transplant recipients receiving low-dose alemtuzumab for GvHD prophylaxis we found a low frequency of viral reactivations and no association of $\mathrm{BKPyV}$ or JCPyV with severe viral infections or GvHD. BKPyV-associated hemorrhagic cystitis is significantly correlated with toxoplasmosis in this population. Further prospective studies with drug and immune system monitoring are necessary to elucidate the link of polyomavirus BK and JC to other transplant-associated infections like CMV reactivation. Even the development of a clinical model system might be helpful to improve patient care.

\section{Disclosure Statement}

The authors of this work have nothing to disclose and no conflict of interests. \begin{tabular}{ll}
\hline Acta Haematol 2017;138:3-9 \\
DOI: $10.1159 / 000468972$
\end{tabular}
Schneidewind/Neumann/Knoll/ Zimmermann/Smola/Schmidt/Krüger 


\section{References}

1 Coppo P, Laporte JPH, Aoudjhane M, Lebon P, Isnard F, Lasage S, Gorin NC, Najman A: Progressive multifocal leukoencephalopathy with peripheral demyelinating neuropathy after autologous bone marrow transplantation for acute myeloblastiv leukemia (FAB5). Bone Marrow Transplant 1999;23:401-403.

2 Bogdanovic G, Priftakis P, Giraud G, Kuzniar M, Ferraldeschi R, Kokhaei P, Mellstedt H, Remberger M, Ljungman P, Winiarski J, Dalianis $\mathrm{T}$ : Association between high $\mathrm{BK}$ virus load in urine samples of patients with graftversus-host disease and development of hemorrhagic cystitis after hematopoietic stem cell transplantation. J Clinical Microbiol 2004;42: 5394-5392.

3 Toyoda M, Puliyanda DP, Amet N, Baden L, Cam V, Radha R, Pao A, Vo A, Bunnapradist S, Moudgil A, Jordan SC: Co-infection of polyomavirus-BK and cytomegalovirus in renal transplant recipients. Transplantation 2005;27:198-205.

4 Daibata M, Hatakeyama N, Kamioka M, Nemoto Y, Hiroi M, Miyoshi I, Taguchi H: Detection of human herpesvirus 6 and JC virus in progressive multifocal leukoencephalopathy complicating follicular lymphoma. Am J Hematol 2001;67:200-205.

5 Abudayyeh A, Hamdi A, Lin H, Abdelrahim M, Rondon G, Andersson BS, Afrough A, Martinez CS, Tarrand JJ, Kontoyiannis DP, Marin D, Gaber AO, Salahudeen A, Oran B, Chemaly RF, Olson A, Jones R, Popat U, Champlin RE, Shpall EJ, Winkelmayer WC, Rezvani K: Symptomatic BK virus infection is associated with kidney function decline and poor survival in allogeneic hematopoietic stem cell recipients. Am J Transplant 2016;16: 1492-1502.
6 Gilis L, Morisset S, Billaud G, Ducastelle-Lepretre S, Labussiere-Wallet $\mathrm{H}$, Nicolini FE, Barraco F, Detrait M, Thomas X, Tedone N, Sobh M, Chidiac C, Ferry T, Salles G, Michallet M, Ader F; Lyon BK Virus Study Group High burden of BK virus-associated hemorrhagic cystitis in patients undergoing allogeneic hematopoietic stem cell transplantation. Bone Marrow Transplant 2014;49:664-670.

7 Peleg AY, Husain S, Kwak EJ, Silveira FP, Ndirangu M, Tran J, Shutt KA, Shapiro R, Thai N, Abu-Elmagd K, McCurry KR, Marcos A, Perterson DL: Opportunistic infections in 547 organ transplant recipients receiving alemtuzumab, a humanized monclonal CD52 antibody. Clin Infect Dis 2007;44:204-212.

8 Quintela A, Escuret V, Roux S, Bonnafous P, Gilis L, Barraco F, Labussiere-Wallet H, Ducastelle-Lepretre S, Nicolini FE, Thomas X, Chidiac C, Ferry T, Frobert E, Morisset S, Poitevin-Later F, Monneret G, Michallet M, Ader F; Lyon HEMINF Study Group: HHV-6 infection after allogeneic hematopoietic stem cell transplantation: from chromosomal integration to viral co-infections and T-cell reconstitution patterns. J Infect 2016;72:214222 .

9 Watcharananan SP, Kiertiburanakul S, Piyatuctsanawong W, Anurathapan U, Sungkanuparph S, Pakakasama S, Chanttratita W, Hongeng S: Cytomegalovirus, adenovirus, and polyoma co-infection among pediatric recipients of allogeneic stem cell transplantation: characteristics and outcome. Pediatr Transplant 2010;14:675-681.
10 Satyanarayana G, Marty FM, Tan CS: The polyomavirus puzzle: is host immune response beneficial in controlling BK virus after adult hematopoietic cell transplantation? Transpl Infect Dis 2014;16:521-531.

11 Peterson L, Ostermann H, Fiegl M, Tischer J, Jaeger G, Rieger CT: Reactivation of polyomavirus in the genitourinary tract is significantly associated with severe GvHD and oral mucositis following allogeneic stem cell transplantation. Infection 2016;44:483-490.

12 Tong A, Flemming K, McInnes E, Oliver S, Craig J: Enhancing transparency in reporting the synthesis of qualitative research: ENTREQ. BMC Med Res Methodol 2012;12: 181-188.

13 Uhm J, Hamad N, Michelis FV, Shanavas M, Kuruvilla J, Gupta V, Lipton JH, Messner HA, Seftel M, Kim DD: The risk of polyomavirus BK-associated hemorrhagic cystitis after allogeneic hematopoietic SCT is associated with myeloablative conditioning, CMV viremia and severe acute GvHD. Bone Marrow Transplant 2014;49:1528-1534.

14 Elfadawy N, Flechner SM Liu X, Schold J, Srinivas TR, Poggio E, Fatica R, Avery R, Mossad SB: CMV viremia is associated with a decreased incidence of BKV reactivation after kidney and kidney-pancreas transplantation. Transplantation 2013;96:1097-1103.

15 Hayden RT, Gu Z, Liu W, Lovins R, Kasow K, Woodard P, Srivastava K, Leung W: Risk factors for hemorrhagic cystitis in pediatric allogeneic hematopoietic stem cell transplant recipients. Transplant Infect Dis 2015;17: 234-241.

16 Schneidewind L, Neumann T, Burchardt M, Krüger W: Urological complications and BK virus associated hemorrhagic cystitis under allogenic stem cell transplantation. Urol Int 2016;97:434-439. 